\title{
Interval of time uncertainty in visual detection'
}

The effects of increasing the length of an interval of continuous temporal uncertainty (ITU) on the detectability of visual signals were investigated in a 'Yes/No' detection situation. Ss were uncertain about when a signal might occur within a given observation interval, the duration of which was varied. Longer intervals of uncertainty resulted in a decrement in detectability, which was shown to be directly attributable to increased false alarm rates. It was suggested that observers have more opportunities for confusing signals with noise. The time course of detectability within a given ITU was also investigated, but there were no significant variations.

In a signal detection situation, an ideal observer requires full knowledge of the signal parameters in order to achieve optimum performance. Several studies have shown that when uncertainty is introduced about one or more parameters of the signal situation, there is usually some decrement in performance. Most of this work has been done on the detection of auditory signals. Creelman (1960), for instance, introduced frequency uncertainty and found a decrement in detection. He has postulated a "multiple-filter" model, suggesting that observers "test" for each possible signal frequency in each observation interval of a two aiternative forced-choice trial. The number of incorrect decisions is likely to increase in this "multiple-test" for signal frequency. Results of experiments tend, on the whole, to support this model, although the variability of the data makes any simple conclusion dubious. Veniar (1958) extended the number and range of possible signals in the frequency uncertainty situation. Again, it is difficult to summarize the results because of considerable scatter in the data. Although the decrement in detection arising from signal uncertainty tends to increase with the frequency separation of the posaible signals, it is unlikely to be a statistically significant effect. Indeed, for two out of four $\mathrm{Ss}$, the data fit the null hypothesis more closely.

Green (1961), in fact, refers to these apparently inconclusive results of Creelman and Veniar, and, from the data of his own experiments, suggests that one should expect very little decrease in detectability from signal frequency uncertainty, even in extreme conditions, since there may already be a considerable amount of initial uncertainty about signal parameters with fixed and specified signals.

An interesting experiment by Shipley (1959) supports indirectly Green's assumption. In a 2-Alt FC detectability experiment, a strong and a weak signal were presented at random. The proportion of correct responses was higher following correct responses to the strong signal than following correct responses to a weak signal. It seems that the strong signal was a more effective cue in reducing uncertainty about signal parameters. Similarly, Gundy (1961) investigated the auditory detection of an unspecified signal, and found that performance was initially at chance level, but gradually Improved as the experiment progressedin contrast to the stable level of performance when the signal was specified.

On the other hand, in a stimulus-response uncertainty situation, Pollack (1959) found that accuracy of message reception was independent of message-source uncertainty, but critically dependent upon the size of the set of relevant response categories. Also, Shipley (1960) suggests that Luce's choice model leads to the prediction that there will be no decrement as a result of uncertainty about frequency if the observer is informed after the observation, but before his response, which frequency was presented. In other words, Pollack and Shipley both postulate a system of "response selection," rather than "perceptual tuning" or filter mechanisms, as the important factor in detection and recognition under conditions of uncertainty.

When investigating the effects of temporal uncertainty on detection, one must distinguish between intervals of continuous time uncertainty (ITU) and discrete temporal observation intervals. The latter are usually found in n-Alt FC experiments, where the signal might occur in any one of ndiscrete temporal intervals. In such situations, one finds that increasing the number of alternative temporal intervals has very little effect on the detectability of an auditory signal (Swets, 1961), or of a visual signal (Howarth \& Lowe, 1966). When intervals of continuous time uncertainty are involved, however, the detectability of an auditory signal is found to decrease with the length of interval (Egan, Schulman, \& Greenberg, 1959, 1961). It is suggested that a substantial part of the decrement in performance may be due to poor memory for the input signal, and that with the longer intervals, there may be more opportuntty for confusing the signal with nolse. If this is so, then the false alarm rate, $P(Y / N)$, should increase with ITU.

The aim of the present experiment was to repeat certain aspects of the study by Egan, Greenberg, and Schulman (1961), using visual, as opposed to auditory, detection to determine whether one would obtain similar decrements in detectability as ITU increases. If so, is the decrement due to an increase in the false alarm rate, as suggested above? 


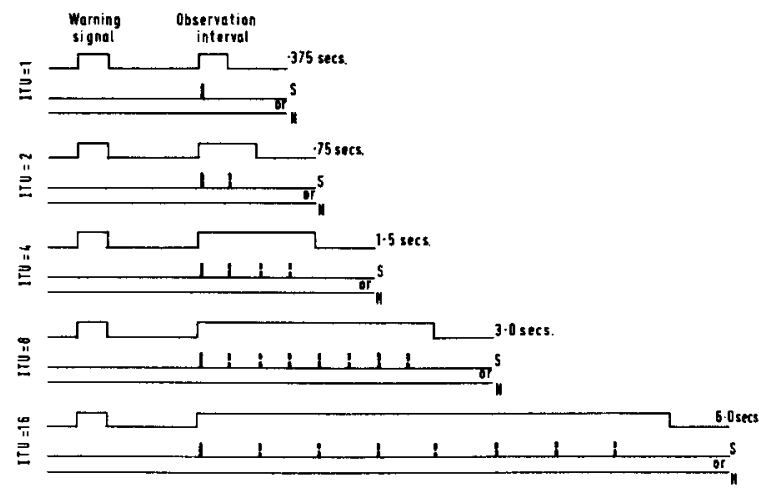

Fig. 1. Schema of sequence of events.

A secondary aim of the experiment was to observe the time course of detectability. Egan, Greenberg, and Schulman's (1961) study also investigated variability in detection during the course of a given observation interval, to check whether the listener's attention "flagged." They found detectability to be the same throughout the interval. However, they only used one interval ( 2 sec.) and simply compared two detectability measures, depending on whether the signal occurred in the first or second half of the observation interval. In the present experiment, it was decided to investigate this time course of detectability more closely, and detection measures could be obtained for all signal occurrence times within every ITU.

\section{Method}

Ss were positioned $15 \mathrm{ft}$. away from a large screen (9 ft. $x 8 \mathrm{ft}$. ), in the center of which was a background circle of moderately low intensity, displayed by a projection unit in the E's cubicle. At the center of this was a small, red fixation light. The stimulus was a very brief ( 1 msec.) circular light flash from an externally triggered stroboscope, and was directed through an optical projection system to a spot on the screen about $7^{\circ}$ from the fixation point. Warning signals and observation intervals were defined by an auditory tone through a loudspeaker. Intensity control was by means of a switching system, utilizing Kodak neutral density filters, via a uni-selector programming unit, which also controlled ITU duration, time of signal occurrence, and the auditory warning signal. Responses were made by pressing one of two keys-'Tes" or "No." There was fairly low, even illumination throughout the observation room, and the $E$ and apparatus were positioned inside a cubicle within this room.

Observers were introduced to the situation and presented with examples of the stimulus. A few practice sessions followed. In the experimental trials, the sequence of events was as depicted in Fig. 1. For ITU 1 , the interval was of 0.375 sec. duration, and there was only one possible occurrence time for the signal. For ITU $2\left(0.75 \mathrm{sec}_{\text {. }}\right)$ and ITU $4(1.5 \mathrm{sec}$.$) , the signal$ occurred randomly at any one of two or four, respectively, possible stimulus times within the interval. For ITU 8 and ITU 16, the signal could occur randomly at any one of eight possible times. In all cases, the probability of a signal occurring equalled the probability of there being no signal, i.e., $p(S)=p(N)=0.5$. The experimental session was divided into blocks of about 30 trials with rest pauses in between. The duration of the ITU was fixed for any one block of trials, but varied systematically from one block to another. The observer was always informed of the particular ITU being given.

The Ss were two male graduates, who we :e experienced in making judgments in signal detectior. tasks. For $S 1$, the maximum ITU was $3 \mathrm{sec}$., and the maximum number of trials for each ITU was 96 ; for S 2, ITUs were extended up to $6 \mathrm{sec}$., and the maximum number of trials for each ITU was 240 .

\section{Results and Discussion}

Figure 2 shows some systematic variation in detectability with ITU. But although the data suggest that uncertainty regarding the time of onset of the signal affects performance, they do not show complete agreement with the results of Egan et al (1961), who found a definite progressive decrement as ITU increased. The present data suggest that conditions of minimum temporal uncertainty (ITU 1) do not result in optimum performance. An interval of 0.75 sec. (ITU 2) shows the highest detectability scores for both signal intensities and both Ss. ITUs of longer duration, however, result in a progressive decrement in performance, similar to the relationship between $d^{\prime}$ and ITU shown by Egan et al (1961).

It was thought that this rather surprising decrement under ITU 1 was possibly an artifact of the experimental setup. In Condition ITU 1, the signal occurred almost immediately after the commencement of the observation interval, indicated by an auditory tone (see Fig. 1). Thus, subjectively, it might appear as

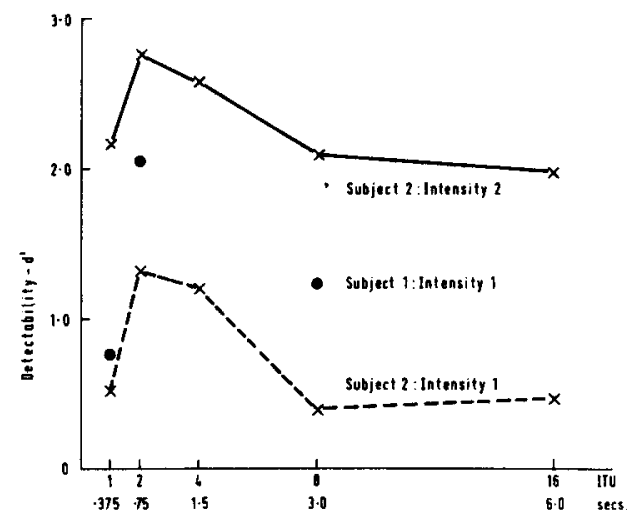

Fig. 2. Detectability (d') as a function of ITU, (d' for $S 1$, Intensity 2 , ITU 4, was not calculable from the data). 
if the beginning of the auditory tone indicating the observation interval and the visual signal occurred at about the same time. The warning signal came $1.5 \mathrm{sec}$. before the ITU, so one possibility might be that the $S$ was faced with an appreciable amount of temporal uncertainty, even in this condition. If this was so, however, one would also expect signals occurring at presentation Time 1 within the longer ITUs to have significantly lower probabilities of detection. The data can be examined to test this hypothesis. Table 1 shows, however, that this is not the case. Signals occurring at presentation Time 1 are detected just as well as at other times. For $S 1$, the average $P(Y / S)$ for presentation Time 1 was 0.18 , for ITUs greater than ITU 1 , as opposed to an average $P(Y / S)$ of 0.17 for all other presentation times; for $S 2, P(Y / S)$ was 0.60 and 0.55 , respectively.

One result which is very clearly indicated by the data, however, is that concerning the variation in false alarm rate as a function of ITU (Fig. 3). If one compares these data with the detectability data of Fig. 2, it is quite obvious that the variation in $d^{\prime}$ as a function of ITU is directly attributable to the variation in false alarm rate $(P(Y / N))$, and not to variation in detection $(\mathrm{P}(\mathrm{Y} / \mathrm{S}))$. In other words, temporal uncertainty in this type of situation affects the detectability of signals by increasing the number of false alarms (or false postive responses). This, of course, is consistent with the hypothesis of Egan et al (1961) that, with increased temporal uncertainty, there is more opportunity for confusing signals with noise.

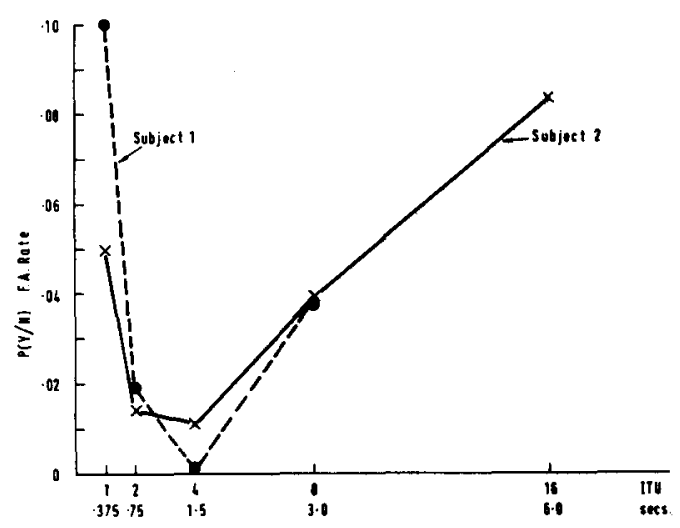

Fig. 3. False alam rate as a function of ITU.
Table 1. Probability of detection as a function of time of signal occurrence within ITU.

\begin{tabular}{ccccccccccc} 
Subject & ITU & secs. & 1 & 2 & 3 & 4 & 5 & 6 & 7 & 8 \\
\hline 1 & 1 & .375 & .15 & & & & & & & \\
& 2 & .75 & .38 & .17 & & & & & & \\
& 4 & 1.5 & .17 & .08 & .17 & .25 & & & & \\
& 8 & 3.0 & 0 & 0 & .33 & .17 & .50 & .17 & 0 & 0 \\
$I 1$ & 1 & .375 & .56 & & & & & & & \\
& 2 & .75 & .60 & .52 & & & & & & \\
& 4 & 1.5 & .55 & .43 & .52 & .62 & & & & \\
& 8 & 3.0 & .44 & .55 & .61 & .50 & .61 & .50 & .53 & .55 \\
& 16 & 6.0 & .80 & .47 & .47 & .67 & .80 & .67 & .60 & .60 \\
\hline
\end{tabular}

\section{References}

Creelman, C. D. Detection of signals of uncertain frequency. $J$. Acoust. Soc. Amer., 1960, 32, 775-810.

Egan, J. P., Greenberg, G. Z., Schulman, A. I. Detection of signals presented at random times. J. Acoust. Soc. Amer., 1959, 31, 1579 (Abstract).

Egan, J. P., Greenberg, G. Z., \& Schulman, A. I. Interval of time uncertainty in auditory detection. J. Acoust. Soc. Amer., 1961, 33, 771-778.

Egan, J. P., Schulman, A. I., \& Greenberg, G. Z. Memory for waveform and time uncertainty in auditory detection. J. Acoust. Soc. Amer., 1961, 33, 779-781.

Green, D. M. Detection of auditory sinusoids of uncertain frequency. J. Acoust. Soc. Amer., 1961, 33, 897-903.

Gundy, R. F, Auditory detection of an unspecified signal. $J$. Acoust. Soc. Amer., 1961, 33, 1008-1012.

Howarth, C. I., \& Lowe, G. Statistical detection theory of Piper's law. Nature, 1966, 212, 324-326.

Pollack, I. Message uncertainty and message reception. J. Acoust. Soc. Amer., 1959, 31, 1500-1508.

Shipley, E. F. Cueing as a determiner of apparent variability in sensitivity. J. Acoust. Soc. Amer., 1959, 31, 834.

Shipley, E. F. A model for detection and recognition with signal uncertainty. Psychometrika, 1960, 25, 273-289.

Swets, J. A. Indices of signal detectability obtained with various psychophysical procedures. J. Acoust. Soc. Amer., 1959, 31, 511-513.

Veniar, F, A. Signal detection as a function of frequency ensemble. J. Acoust. Soc. Amer., 1958, 30, 1020-1024; 1075-1078.

\section{Note}

1. This research was supported by a grant from the Department of Scientific and Industrial Research (now Science Research Council). England, on 'Human decision processes', awarded to Professor C. 1. Howarth, University of Nottingham, England. Appreciation is expressed to A. R. McKellar for assistance at various stages of the project.

(Accepted for publication March 9, 1967.) 